\title{
A feasibility, randomised controlled trial of a complex breathlessness intervention in idiopathic pulmonary fibrosis (BREEZE-IPF): study protocol
}

\author{
Caroline Wright ${ }^{1}$, Simon P. Hart (10 ${ }^{1}$, Victoria Allgar ${ }^{2}$, Anne English $^{3}$, Flavia Swan $^{4}$, \\ Judith Dyson ${ }^{5}$, Gerry Richardson ${ }^{6}$, Maureen Twiddy ${ }^{5}$, Judith Cohen ${ }^{7}$, \\ Jamilla Hussain ${ }^{4}$, Miriam Johnson ${ }^{4}$, Ian Hargreaves ${ }^{1}$ and Michael G. Crooks ${ }^{1}$
}

\section{ABSTRACT}

Introduction: Idiopathic pulmonary fibrosis (IPF) is a chronic and progressive lung disease that causes breathlessness and cough that worsen over time, limiting daily activities and negatively impacting quality of life. Although treatments are now available that slow the rate of lung function decline, trials of these treatments have failed to show improvement in symptoms or quality of life. There is an immediate unmet need for evidenced-based interventions that improve patients' symptom burden and make a difference to everyday living. This study aims to assess the feasibility of conducting a definitive randomised controlled trial of a holistic, complex breathlessness intervention in people with IPF.

Methods and analysis: The trial is a two-centre, randomised controlled feasibility trial of a complex breathlessness intervention compared with usual care in patients with IPF. 50 participants will be recruited from secondary care IPF clinics and randomised 1:1 to either start the intervention within 1 week of randomisation (fast-track group) or to receive usual care for 8 weeks before receiving the intervention (wait-list group). Participants will remain in the study for a total of 16 weeks. Outcome measures will be feasibility outcomes, including recruitment, retention, acceptability and fidelity of the intervention. Clinical outcomes will be measured to inform outcome selection and sample size calculation for a definitive trial.

Ethics and dissemination: Yorkshire and The Humber - Bradford Leeds Research Ethics Committee approved the study protocol (REC 18/YH/0147). Results of the main trial and all secondary end-points will be submitted for publication in a peer-reviewed journal.

@ERSpublications

Idiopathic pulmonary fibrosis (IPF) is a chronic and progressive lung disease. This study protocol describes the BREEZE-IPF study: a feasibility, randomised controlled trial of a holistic, complex breathlessness intervention in IPF. http://bit.ly/33eF9im

Cite this article as: Wright $\mathrm{C}$, Hart SP, Allgar V, et al. A feasibility, randomised controlled trial of a complex breathlessness intervention in idiopathic pulmonary fibrosis (BREEZE-IPF): study protocol. ERJ Open Res 2019; 5: 00186-2019 [https://doi.org/10.1183/23120541.00186-2019].

This study is registered at the ISRCTN registry with identifier number ISRCTN13784514. The investigators will agree a data management and access plan with the funder, the National Institute of Health Research, prior to completion of data collection. Anonymised data will be deposited in a discoverable data registry following publication of the primary study outputs. A study data access committee will review requests to access data and will require a data use agreement to be signed. The data use agreement will detail the agreed use and appropriate management of the research data to be shared, and include a requirement for recognition of the contribution of the researchers who generated the data and the original funder.

Received: 26 July 2019 | Accepted: 27 July 2019

Copyright $\odot$ ERS 2019. This article is open access and distributed under the terms of the Creative Commons Attribution Non-Commercial Licence 4.0. 


\section{Introduction}

Idiopathic pulmonary fibrosis (IPF) is a chronic and progressive lung disease with an incidence in the UK of around 8.04 per 100000 person-years [1]. Breathlessness and cough are common symptoms in IPF and typically worsen over time, limiting daily activities and impacting quality of life [2]. The impact of breathlessness on IPF patients can be profound. In addition to the detrimental impact on quality of life, chronic breathlessness is associated with anxiety and depression [3] and reduced survival [4]. The median survival is 3 years from diagnosis and until recently there were no disease-modifying therapies [5]. Two therapies are now available that slow the rate of lung function decline $[6,7]$; however, trials have failed to show improvement in symptoms or quality of life [6,7]. This emphasises the importance of a holistic approach to patient management, and the complex interplay between symptoms and disease outcomes. There is therefore an immediate unmet need for evidenced-based interventions that improve patients' symptom burden and make a difference to everyday living.

Pulmonary rehabilitation improves IPF patients' exercise capacity, symptoms and quality of life [8-12]. However, despite featuring in national guidelines, access to programmes varies across the UK and rehabilitation programmes are generally tailored to patients with chronic obstructive pulmonary disease. While pulmonary rehabilitation is a core component of IPF patients' management, it is not suitable for or tolerated by all patients and therefore many remain unable to access evidence-based treatment for their symptoms.

It is recommended that IPF patients should have access to palliative care services to support symptom management. Palliative breathlessness interventions can be pharmacological and/or nonpharmacological. However, their effectiveness in IPF patients is unknown. Nonpharmacological breathlessness interventions have been studied in patients with chronic breathlessness caused by cardiorespiratory diseases, with benefits observed in terms of reduced distress due to breathlessness and increased breathlessness mastery [13-15]. Subgroup analysis revealed patients with nonmalignant diseases who received a breathlessness intervention as part of a randomised controlled trial had prolonged survival [13]. However, patients with interstitial lung disease are under-represented in these studies.

Evidence of the efficacy of palliative care breathlessness strategies in IPF patients is limited [16]. The poor prognosis, variable disease trajectory and absence of any existing, widely accessible, evidence-based treatment to improve patients' symptoms set IPF apart from other causes of chronic breathlessness. Studies in this patient group are needed to help clinicians and commissioners identify the efficacy, reproducibility and cost-effectiveness of an intervention to improve breathlessness management in IPF patients.

Dove House Hospice (Hull, UK) provides a specialist breathlessness service led by an experienced respiratory physiotherapist. Patients attending the service undergo a comprehensive assessment prior to receiving training in four breathlessness management techniques. As part of routine care, patients attending the Hull breathlessness service complete numerical rating scales (NRSs) scored 0-10 before and after attendance to assess the following characteristics of breathlessness: severity, distress and ability to cope. An evaluation of 22 consecutive IPF patients showed significant improvements in terms of average breathlessness in the past $24 \mathrm{~h}$ (NRS change: $-0.27,95 \% \mathrm{CI}-0.52--0.03$; $\mathrm{p}=0.03$ ), worst breathlessness in the past $24 \mathrm{~h}$ (NRS change: $-1.05,95 \% \mathrm{CI}-1.47--0.6$; $\mathrm{p}<0.01$ ), distress caused by breathlessness (NRS change: $-0.95,95 \% \mathrm{CI}-1.21--0.61 ; \mathrm{p}<0.01)$ and perceived ability to cope with breathlessness (NRS change: $1.00,95 \%$ CI $0.68-1.32$; $\mathrm{p}<0.01$ ) [17]. A similar breathlessness intervention has been studied in a multicentre trial in malignant disease, demonstrating that the breathlessness intervention can effectively be implemented across different centres [15]. The scalability of this breathlessness intervention and preliminary data suggesting benefit in an IPF cohort led us to select this intervention for this trial.

As large-scale trials of a complex breathlessness intervention have not been attempted in this patient group to date, the BREEZE-IPF study is designed to assess the feasibility of conducting a future definitive study.

Affiliations: ${ }^{1}$ Respiratory Research Group, Hull York Medical School, Cottingham, UK. ${ }^{2}$ Health Sciences, University of York, York, UK. ${ }^{3}$ Dove House Hospice Palliative Care Physiotherapy Team, NHS Humber Foundation Trust, Willerby, UK. "Wolfson Palliative Care Research Group, Hull York Medical School, Cottingham, UK. ${ }^{5}$ Institute of Clinical and Applied Health Research, University of Hull, Kingston upon Hull, UK. ${ }^{6}$ Centre for Health Economics, University of York, York, UK. ${ }^{7}$ Hull Health Trials Unit, University of Hull, Kingston upon Hull, UK.

Correspondence: Michael G. Crooks, Respiratory Research Group, Institute for Clinical and Applied Health Research, Hull York Medical School, Castle Hill Hospital, Cottingham, HU16 5JQ, UK.

E-mail: michael.crooksanhs.net 


\section{Study aim and objectives}

The BREEZE-IPF study aims to address the following areas of uncertainty relating to the design and conduct of a definitive, phase 3, randomised controlled trial of a holistic complex breathlessness intervention in people with IPF.

\section{Recruitment}

We will identify the eligibility/consent ratio, recruitment rate and participant retention rate to inform the number of sites needed to enrol sufficient participants within an acceptable timeline. We will assess acceptability of recruitment, randomisation and trial processes from the patients' perspective.

\section{Intervention}

We will assess the acceptability and fidelity of the intervention by measuring adherence in delivery and uptake, and via patient and carer interviews. This will include documenting aspects of breathlessness interventions used in the control arm provided ad hoc, e.g. breathing techniques and use of a hand-held fan.

\section{Data quality}

We will assess the amount and pattern of missing data for study measures and completion of questionnaires and other study procedures at each time-point. Data variability across the range of outcome measures will also be assessed. These findings will inform the choice of primary and secondary outcomes for the definitive trial.

\section{Outcomes}

We will determine which is the most appropriate primary outcome and agree other study measures for the definitive trial by identifying: 1) patient views about relevance, importance and acceptability of questionnaires or other measures (qualitative interview data); 2) data completion; and 3) data variability for potential primary outcome measures to inform sample size calculation for the definitive trial.

\section{Health economic assessment}

We will assess the feasibility of collecting the data required to undertake a cost-effectiveness analysis in the definitive trial.

\section{Methods and analysis \\ Design}

The trial is a feasibility, wait-list design, randomised controlled trial of a complex breathlessness intervention compared with usual care in patients with IPF and chronic breathlessness. Participants will be randomised 1:1 to start the intervention within 1 week of randomisation (fast-track group) or to receive usual care for 8 weeks before receiving the intervention (wait-list group). Participants will remain in the study for 16 weeks. A wait-list design was selected to ensure that all participants will be able to access the breathlessness intervention during the study. The wait-list period was chosen based on feedback from patients, their carers and healthcare professionals.

This feasibility trial is being conducted in two centres to reflect the different clinical environments where IPF patients are managed in the UK. One site is a specialist interstitial lung disease centre and the other centre is a rural district general hospital. The first patient was randomised in August 2018, with a recruitment target of 50 patients over a period of 18 months.

The trial design and procedures are outlined in the study flowchart (figure 1) and schedule of events (table 1).

\section{Study population}

Participants will be identified through participating site outpatient departments, interstitial lung disease multidisciplinary team (MDT) meetings and research databases. Clinicians working in study sites will be trained in the study protocol, including inclusion and exclusion criteria.

Eligible patients will be aged $\geqslant 50$ years, have IPF diagnosed by an MDT and experience chronic breathlessness (defined as modified Medical Research Council grade 3 or 4 breathlessness that persists despite optimal treatment of the underlying pathophysiology and is causing disability [18]). Participants are required to have oxygen saturations of $\geqslant 90 \%$ breathing room air or on their usual flow rate of supplemental oxygen.

Participants will be considered unable to take part if they: 1) have another significant cardiorespiratory disease considered to be the primary cause of their breathlessness; 2) have completed pulmonary rehabilitation or attended a breathlessness clinic within 3 months of enrolment; or 3) are unable or unwilling to consent or complete study procedures. 


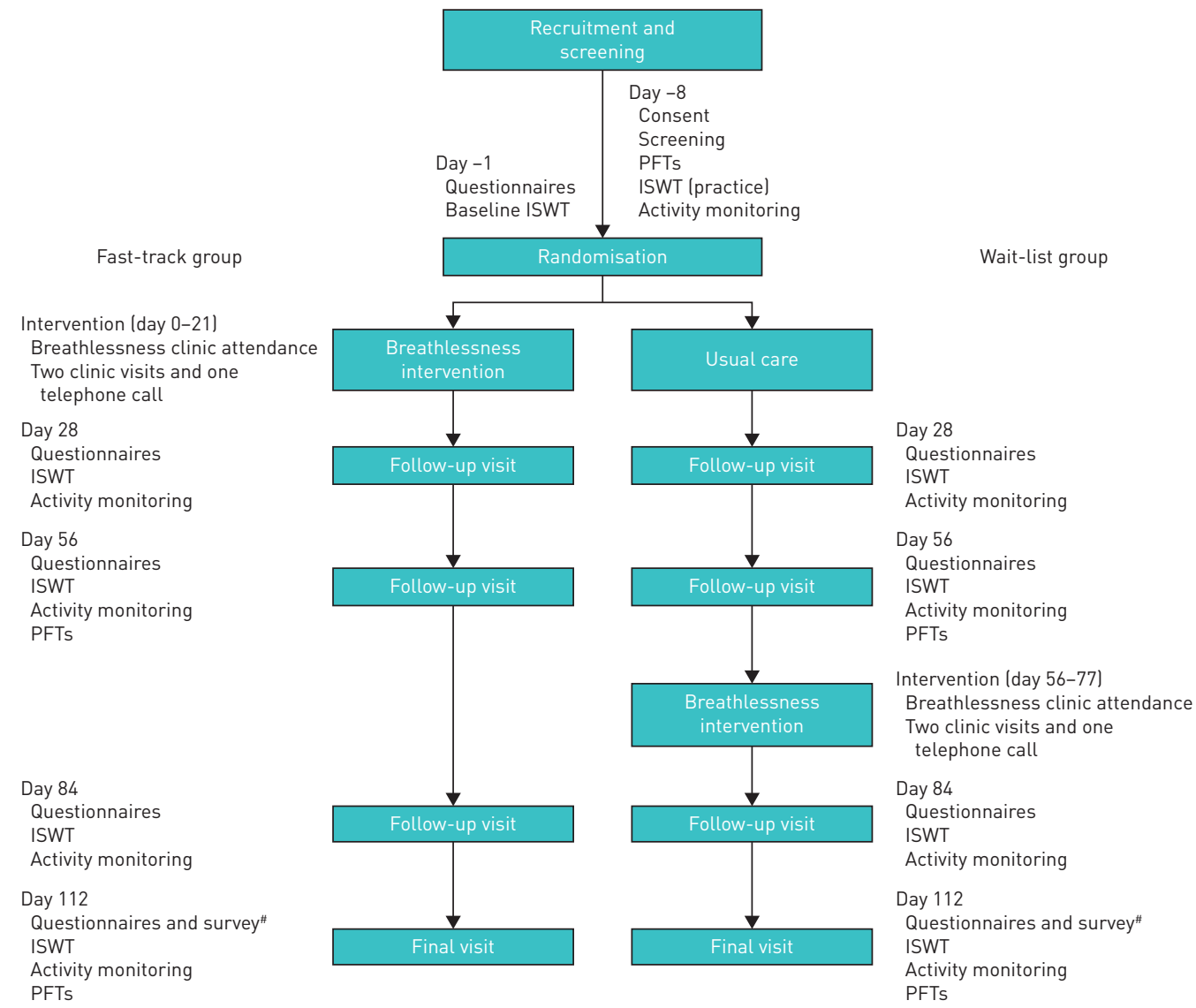

FIGURE 1 Feasibility wait-list study flowchart. PFT: pulmonary function test; ISWT: incremental shuttle walk test. \#: a study experience survey will be sent to participants on completion of the study or, if participants discontinue the study early, as soon after withdrawal as practical.

\section{Sample size}

Since this is a feasibility study, a formal sample size calculation has not been performed. We will recruit 50 patients over 18 months, 25 in each arm, which will be sufficient to assess the feasibility parameters.

\section{Recruitment and consent}

Participants will be identified by their usual care teams and provided with information about the study. Potential participants will be given time to consider before agreeing to screening. Randomisation will occur after eligibility is confirmed and informed consent is obtained.

\section{Randomisation and blinding}

Randomisation will be at the individual level using a commercial web-based randomisation system. Participants will be randomised in a 1:1 ratio using random permuted blocks, fast-track intervention to wait-list intervention, stratified by site. The randomisation sequence will be prepared by an independent statistician from the Hull Health Trials Unit; all trial staff and sites will remain blinded to the sequence. It is not possible to blind either patients, clinicians or trial staff to the treatment group allocation.

\section{Intervention}

The breathlessness intervention is delivered during three consultations with a trained practitioner (physiotherapist, nurse specialist and/or occupational therapist). The first two consultations are conducted face-to-face, last $\sim 1 \mathrm{~h}$ and take place 1 week apart. A final telephone consultation is conducted 1 week later lasting 10-15 min. The intervention is holistic and is therefore tailored by the therapist to address the patient's individual needs. However, the following core aspects of breathlessness management will be delivered to all participants: 1) breathing control techniques; 2) instructions on using a hand-held fan (a fan will be provided); 3) pacing and breathlessness management during everyday activities, including positions for recovery from exertional breathlessness and information on the importance of exercise; and 4) techniques to promote relaxation and manage anxiety and panic. 


\section{TABLE 1 Schedule of events}

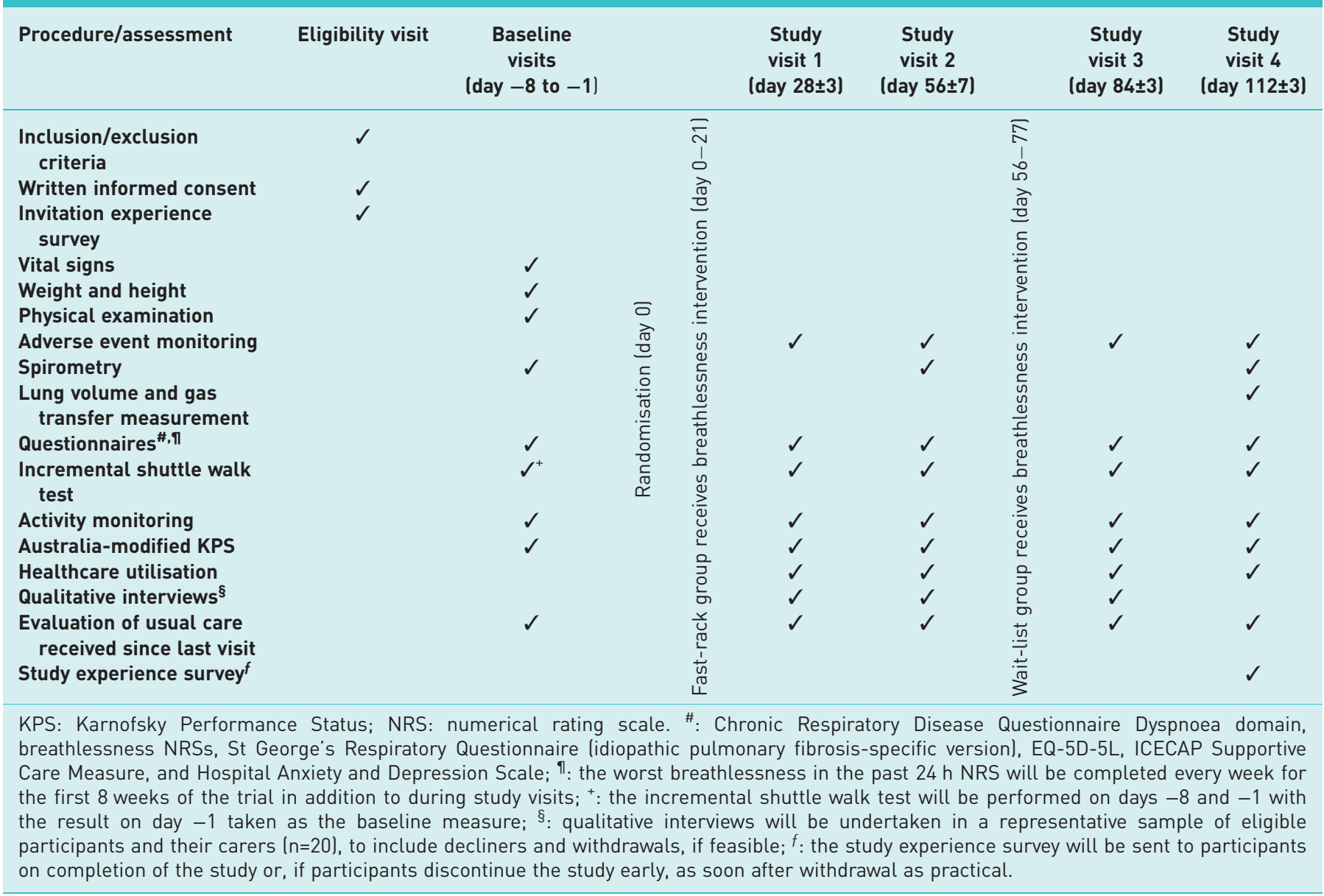

All participants will be given bespoke breathlessness intervention leaflets (based on the information leaflets created by the Breathlessness Intervention Service at Addenbrooke's Hospital, Cambridge, UK, with permission) that cover the following breathlessness management techniques: 1) the hand-held fan; 2) breathing control and techniques to ease breathlessness; 3) positions to ease breathlessness; 4) managing thoughts about breathlessness; 5) relaxation; 6) conserving energy levels; and 7) exercise.

Delivery of the core components of the breathlessness intervention and provision of the breathlessness leaflets will be recorded to ensure intervention fidelity.

\section{Outcomes and assessments}

The following three feasibility outcomes will be assessed.

1) Recruitment: eligibility to consent ratio, recruitment rate and retention/follow-up rates at each time-point: 4, 8, 12 and 16 weeks.

2) Data quality: completion of clinical outcomes (questionnaires and other assessments) at each time-point (baseline, 4, 8, 12 and 16 weeks) and patterns of missing data for the study measures.

3) Intervention: adherence in delivery and uptake will be documented in the clinical record; acceptability will be assessed during interviews.

Additionally, a series of clinical outcomes will be measured to inform outcome selection and sample size calculation for a definitive trial. Clinical outcome measures will evaluate a range of domains.

\section{Breathlessness}

Breathlessness mastery will be assessed using the breathlessness mastery domain of the Chronic Respiratory Disease Questionnaire [19]. NRSs will be used to investigate the following aspects of breathlessness over the past $24 \mathrm{~h}$ : best breathlessness over the past $24 \mathrm{~h}$, worst breathlessness over the past 
$24 \mathrm{~h}$, distress caused by breathlessness over the past $24 \mathrm{~h}$ and coping with breathlessness over the past $24 \mathrm{~h}$. The NRSs are scored $0-10$, where 0 equates to no breathlessness and 10 is the worst possible breathlessness.

\section{Quality of life}

The St George's Respiratory Questionnaire for patients with IPF will be used to measure disease-specific quality of life. The EuroQol EQ-5D five-level version (EQ-5D-5L) and EQ-5D visual analogue scale (EQ-5D-VAS) will be used to measure generic health-related quality of life.

Mood

The Hospital Anxiety and Depression Scale will be used to calculate anxiety and depression scores.

Physical activity, functional status and exercise capacity

Australian-modified Karnofsky Performance Status will be measured to assess functional status.

Exercise capacity will be assessed using the incremental shuttle walk test (performed in accordance with European Respiratory Society/American Thoracic Society technical standards [20]). A practice incremental shuttle walk test will be performed prior to baseline assessment to eliminate the known learning effect.

Physical activity will be measured objectively during normal daily life by asking participants to wear an accelerometer (GT3XP-BT; ActiGraph, Pensacola, FL, USA) using an ankle strap for a period of 7 days. Data on step counts, physical activity duration and physical activity levels will be evaluated.

\section{Pulmonary function}

Pulmonary function tests will be conducted in accordance with American Thoracic Society/European Respiratory Society guidelines [21] at baseline and at intervals throughout the study (table 1). Lung function is being measured to assess the number of patients with progressive disease during the trial to inform whether this needs to be accounted for in the statistical plan for the substantive trial.

\section{Health economics}

Health service utilisation will be recorded, including: general practitioner attendance, practice nurse attendance, outpatient appointment attendance (consultant), specialist nurse review (outpatient or home visit), emergency department attendance, hospital admission and hospice admission. EQ-5D-5L, EQ-5D-VAS and ICECAP Supportive Care Measure (ICECAP-SCM) data will be collected. EQ-5D-5L data will allow the calculation of quality-adjusted life-years in a subsequent definitive trial.

\section{Participant feedback}

An invitation experience survey will be used to capture participants' experience of the recruitment process and evaluate reasons for individuals agreeing or declining involvement.

A study experience survey will be given to participants following completion or withdrawal from the study to capture their overall experience of the study and reasons for withdrawal if applicable.

\section{Qualitative substudy}

A series of in-depth semistructured interviews will be performed to establish the perception of patients and their carers about the acceptability of trial processes, study participation, the intervention and outcome measures. Face-to-face interviews will be conducted with a purposive sample of participants (patients and their carers). Focus groups will also be conducted with clinical team members at each site to investigate their experience of the trial processes and the breathlessness intervention.

\section{Data management}

The main study database will be developed by the Respiratory Clinical Trials Unit at Castle Hill Hospital (Cottingham, UK), using the commercial electronic data capture system Medrio (medrio.com). The system uses validation and verification features that will be used to monitor study data quality, in line with the study monitoring plan. A study monitoring plan will be developed by the sponsor quality assurance team, in conjunction with Hull Health Trials Unit, who will assist with monitoring of the study.

\section{Data analysis}

The trial, including the flow of individual participants through each stage, will be reported in accordance with the CONSORT (Consolidated Standards of Reporting Trials) 2010 statement extension to pilot and feasibility trials [22]. 
For the primary outcomes, the feasibility criteria will be recruitment rate, retention rate, adverse events, compliance and acceptability of the intervention. The recruitment rate, consisting of the eligibility and consent rate, will be calculated with $95 \%$ confidence intervals. A table showing baseline demographic and clinical characteristics for each group will be presented to indicate any differences between groups. Patient characteristics will be summarised using appropriate statistics. Median (interquartile range (IQR)) will be reported for ordinal data, mean (standard deviation) will be reported for continuous data and raw count (number (percentage)) will be reported for nominal data.

For patient clinical outcome data, descriptive statistics will be reported: mean (standard deviation) for continuous outcomes, median (IQR) for ordinal data and raw count (number (percentage)) for categorical outcomes. Descriptive statistics will be presented for each group at each time-point: baseline, 4, 8, 12 and 16 weeks. The effect sizes will be calculated with $95 \%$ confidence intervals at each time-point to compare groups. These effect sizes will be used to inform the selection of the primary end-point, primary outcome measure and the sample size (power calculation) for the definitive randomised controlled trial.

Safety data will be summarised using descriptive statistics.

We will present health-related resource use data and health-related quality of life (EQ-5D-5L) and wellbeing outcomes (ICECAP-SCM) for the treatment and control groups. Unit costs of health-related resource use will be presented using published national unit cost estimates. We will generate incremental costs and effects by comparing the treatment and control arms. Within the funding envelope available we will not be able to conduct a full cost-effectiveness analysis using probabilistic sensitivity analysis. Therefore, assessment of the value of conducting further research (using value of information techniques) will not be possible. Instead, we will consider the results of the feasibility work and the potential impact on costs and effects on wellbeing and health-related quality of life that might be shown in a larger trial.

Quantitative and qualitative data will be synthesised to address questions regarding optimal trial delivery and most relevant primary outcome for a subsequent phase 3 trial.

\section{Patient and public involvement}

The research question was developed with input from the Hull Pulmonary Fibrosis Support Group and members contributed to study design. A patient representative is included on the research team, and patients directly contributed to protocol development and production of study documents, including participant information sheets. A patient representative attends trial management group meetings and study progress is discussed at quarterly Hull Pulmonary Fibrosis Support Group meetings. An end-of-study report will be developed in partnership with the patient representative to allow communication of study results to all participants.

\section{Ethics and dissemination}

\section{Regulatory approvals and trial oversight}

The trial protocol has been reviewed and approved by the Yorkshire and The Humber - Bradford Leeds Research Ethics Committee (REC 18/YH/0147). Hull University Teaching Hospitals NHS Trust is the study sponsor and the Hull Health Trials Unit is supporting trial delivery. A Trial Management Group has been convened to oversee trial delivery and operations. An independent Trial Steering Committee will be set up to provide overall supervision for the project on behalf of the project sponsor and project funder.

\section{Safety considerations and adverse event reporting}

Adverse events are defined in the full study protocol and will be reported in accordance with UK NHS Research Ethics Service Guidelines. Hospital admission and death are common in the studied patient group due to their underlying disease. As such, admission or death related to IPF or related comorbidities will be classified as expected and does not require reporting within $24 \mathrm{~h}$, but will be reported within 14 days.

\section{Dissemination}

Results of this feasibility study will be submitted for presentation in the form of an abstract at regional, national and international conferences. Scientific manuscripts will be prepared and submitted for publication in appropriate peer-reviewed journals. A lay summary will be prepared in partnership with patient and public involvement representatives, and shared with patient groups, charities and study participants.

\section{Discussion}

The results of this feasibility study will inform the planning of a definitive randomised controlled trial through assessing the rates of recruitment, retention, adverse events, compliance and acceptability of the intervention. The results from this study will allow us to determine if progressing to a full-size trial using a 
complex breathlessness intervention in IPF is feasible, how many centres would be required, how the study would need to be structured and which outcome measures would be best employed.

Author contributions: M.G. Crooks, V. Allgar, A. English, S.P. Hart, F. Swan, J. Dyson, M. Johnson, J. Hussain G. Richardson, M. Twiddy and J. Cohen are coapplicants on the grant. M.G. Crooks, V. Allgar, A. English, S.P. Hart, F. Swan, J. Dyson, M. Johnson, G. Richardson, M. Twiddy, J. Cohen, I. Hargreaves and C. Wright assisted in development of the protocol and implementation of the study. M.G. Crooks, C. Wright and J. Cohen drafted the manuscript. All authors read and approved the final manuscript.

Support statement: This paper presents independent research funded by the National Institute for Health Research (NIHR) under its Research for Patient Benefit Programme (PB-PG-1216-20020). The views expressed are those of the author(s) and not necessarily those of the NIHR or the Dept of Health and Social Care. Funding information for this article has been deposited with the Crossref Funder Registry.

Conflict of interest: C. Wright reports grants from the NIHR during the conduct of the study. S.P. Hart reports grants from the NIHR during the conduct of the study; and grants, personal fees and nonfinancial support from Boehringer Ingelheim outside the submitted work. V. Allgar reports grants from the NIHR during the conduct of the study. A. English reports grants from the NIHR during the conduct of the study. F. Swan reports grants from the NIHR during the conduct of the study. J. Dyson reports grants from the NIHR during the conduct of the study. G. Richardson reports grants from the NIHR during the conduct of the study. M. Twiddy reports grants from the NIHR during the conduct of the study. J. Cohen reports grants from the NIHR during the conduct of the study. J. Hussain reports grants from the NIHR during the conduct of the study. M. Johnson reports grants from the NIHR during the conduct of the study. I. Hargreaves reports grants from the NIHR during the conduct of the study. M.G. Crooks reports grants from the NIHR during the conduct of the study.

\section{References}

1 Navaratnam V, Fleming KM, West J, et al. The rising incidence of idiopathic pulmonary fibrosis in the UK. Thorax 2011; 66: 462-467.

2 Swigris JJ, Stewart AL, Gould MK, et al. Patients' perspectives in how idiopathic pulmonary fibrosis affects the quality of their lives. Health Qual Life Outcomes 2005; 3: 1.

3 Johnson MJ, Bland JM, Gahbauer EA, et al. Breathlessness in the elderly during the last year of life sufficient to restrict activity: prevalence, pattern and associated factors. J Am Geriatr Soc 2016; 64: 73-80.

4 Nishiyama $\mathrm{O}$, Taniguchi $\mathrm{H}$, Kondoh $\mathrm{Y}$, et al. A simple assessment of dyspnoea as a prognostic indicator in idiopathic pulmonary fibrosis. Eur Respir J 2010; 36: 1067-1072.

5 Bjoraker JA, Ryu JH, Edwin MK, et al. Prognostic significance of histopathologic subsets in idiopathic pulmonary fibrosis. Am J Respir Crit Care Med 1998; 157: 199-203.

6 King TE Jr, Bradford WZ, Casto-Bernardini S, et al. A phase 3 trial of pirfenidone in patients with idiopathic pulmonary fibrosis. N Engl J Med 2014; 370: 2083-2092.

$7 \quad$ Richeldi L, du Bois R, Raghu G, et al. Efficacy and safety of nintedanib in idiopathic pulmonary fibrosis. $N$ Engl J Med 2014; 370: 2071-2082.

8 Holland AE, Hill CJ, Conron M, et al. Short term improvement in exercise capacity and symptoms following exercise training in interstitial lung disease. Thorax 2008; 63: 549-554.

9 Nishiyama O, Kondoh Y, Kimura T, et al. Effects of pulmonary rehabilitation in patients with idiopathic pulmonary fibrosis. Respirology 2008; 13: 394-399.

10 Vainshelboim B, Oliveira J, Yehoshua L, et al. Exercise training-based pulmonary rehabilitation program is clinically beneficial for idiopathic pulmonary fibrosis. Respiration 2014; 88: 378-388.

11 Gaunard IA, Gomez-Marin OW, Ramos CF, et al. Physical activity and quality of life improvements of patients with idiopathic pulmonary fibrosis completing a pulmonary rehabilitation program. Respir Care 2014; 59: 1872-1879.

12 Jackson RM, Gomez-Marin OW, Ramos CF, et al. Exercise limitation in IPF patients: a randomised trial of pulmonary rehabilitation. Lung 2014; 192: 367-376.

13 Higginson IJ, Bausewein C, Reilly CC, et al. An integrated palliative and respiratory care service for patients with advanced disease and refractory breathlessness: a randomised controlled trial. Lancet Respir Med 2014; 2: 979-987.

14 Farquhar M, Prevost AT, McCrone P, et al. Is a specialist breathlessness service more effective and cost-effective for patients with advanced cancer and their carers than standard care? Findings of a mixed-method randomised controlled trial. BMC Med 2014; 12: 194.

15 Johnson MJ, Kanaan M, Richardson G, et al. A randomised controlled trial of three or one breathing technique training sessions for breathlessness in people with malignant lung disease. BMC Med 2015; 13: 213.

16 Bajwah S, Ross JR, Peacock JL, et al. Interventions to improve symptoms and quality of life of patients with fibrotic interstitial lung disease: a systematic review of the literature. Thorax 2012; 68: 867-879.

17 Douglas L, English A, Obita GP, et al. Breath-taking outcomes: evaluation of a specialist breathlessness clinic. Thorax 2016; 17: P229.

18 Johnson MJ, Yorke J, Hansn-Flaschen J, et al. Towards and expert consensus to delineate a clinical syndrome of chronic breathlessness. Eur Respir J 2017; 49: 1602277.

19 Wijkstra PJ, Tenvergert EM, Van Altena R, et al. Reliability and validity of the Chronic Respiratory Questionnaire (CRQ). Thorax 1994; 49: 465-467.

20 Holland AE, Spruit MA, Troosters T, et al. An official European Respiratory Society/American Thoracic Society technical standard: field walking tests in chronic respiratory disease. Eur Respir J 2014; 44: 1428-1446.

21 Miller MR, Crapo R, Hankinson J, et al. General considerations for lung function testing. Eur Respir J 2005; 26 : 153-161.

22 Eldridge SM, Chan CL, Campell MJ, et al. CONSORT 2010 statement extension to randomised pilot and feasibility trials. $B M J$ 2016; 355: i5239. 